\title{
The Re-Invention of the Integrated Quality Management System towards a Culture of Continuous Improvement
}

\author{
Dr J.N. Pylman \\ School for General and Continuing Education, University of Fort Hare, South Africa \\ Email: jpylman@ufh.ca.za
}

Doi:10.5901/mjss.2014.v5n16p419

\begin{abstract}
The first aim of this article was to understand how IQMS practices and systems promote continuous improvement in South African schools. The second was to propose a framework for the re-invention of the IQMS. The research derives from a study concluded in four public primary schools in the Buffalo City Metropolitan area. School principals, members of the school management team (SMT) and teachers were chosen as the focus of the study. The design was a case study and an interview schedule was used to gather information. The findings revealed that (1) IQMS is understood and practiced as an external intervention, resulting in tension between policy, continuous development and improvement in schools. It appears not to have been internalized as an integrated management system; (2) the participants were ambivalent about the IQMS practices as enablers and not as barriers of continuous improvement. Finally, the external and internal quality assurance practices seemed to have been under managerial and imperatives of compliance and accountability. Given the above findings it can be concluded that if continuous improvement is to be achieved through the IQMS strategy, there is a need to re-invent it such that, embedded in its system and structures is a culture of continuous improvement.
\end{abstract}

Keywords: Quality Assurance; Integrated Quality Management System

\section{Introduction}

The education system is usually the largest 'industry' in a country, generating employment and spending a significant portion of the annual government budget (UNESCO, 1999). Schools are responsible to parents and the community for providing the best programmes and high quality education to their learners. A strong and robust system that delivers quality education is seen as a key source of strength to be retained and capitalized upon.

South Africa is undergoing a paradigm shift which has influenced the measurement of the quality of teaching and learning by the introduction of the Integrated Quality Management System (IQMS). The Integrated Quality Management System (IQMS) is a quality management system consisting of three policies: Development Appraisal System (DAS); Whole School Evaluation (WSE) and Performance Management System (PMS), designed to foster a culture of continuous improvement in schools. The IQMS aims at identifying specific needs of teachers, schools and district offices; providing support for continued growth and development, promoting accountability, monitoring institutions overall effectiveness; and evaluating teachers' performance (ELRC, 2003). The policy further assumes that most teachers recognize the need for, and responsibility to improve themselves professionally (Ministerial Committee Report, 2009). Hence continuing professional development for teachers is located within the IQMS. Teacher professional development remains a recurrent theme in this policy, which is pursued by establishing a plan and culture of continuous improvement in schools. Raising the quality of teacher performance through teacher development programmes is essential to improve the overall performance of the education system (Mestry, Hendricks and Bisschoff, 2009). The implementation of IQMS is mandatory in every public school in South Africa.

Despite the introduction of IQMS as an ambitious strategy to improve the quality and performance management in South African schools, several researchers (Gardner, 2003; De Clercq, 2008; Weber, 2005; Rabichund,2011) question the practicality of IQMS and raise concerns as to whether it is feasible to identify needs, provide support, rate performance and evaluate the entire school, using the same instrument. Although IQMS has been designed and implemented as an integrated quality management system, there seems to be no coherence, interactivity and synergy between the three different programmes. Weber (2005); De Clercq (2008) and Biputh and McKenna (2009) concur with this view and assert that the use of IQMS for development, appraisal and accountability, sends ambivalent messages to school staff who are tempted to use the instrument for the sole purpose of rewards and compliance, instead of continuous improvement and development. Furthermore, according to Harvey (2002), the continuous emphasis on procedural 
elements of quality and 'overly bureaucratic procedures' result in detailed paper trails, which stifle innovative processes, continuous quality improvement and loss of professional autonomy.

Notwithstanding the importance of professional development, creating a culture for continuous improvement in schools could be seriously affected for the following reasons according to Mestry, Hendricks and Bisschoff (2009) who cite the insufficient training of teachers in the field of IQMS, lack of insight into IQMS by facilitators, poor leadership of principals and SMT's and the fact that teachers view IQMS as something that has been enforced on them. Whilst for the Department of Education the main objective with IQMS is to "ensure quality public education for all and to constantly improve the quality of teaching and learning" (ELRC, 2003); this does not materialise in practice, as most educators view IQMS as a bureaucratic paper exercise that they have to comply with, rather than a reflective and developmental process (Rabichund, 2011).

\subsection{Unresolved issues in IQMS}

All studies reviewed seem to take it for granted that the three IQMS policies, Developmental Appraisal, Performance Management System and Whole School Evaluation are integrated, without clarifying mechanisms for integration. This then would point to a gap in knowledge in respect of implementation of IQMS and its aims towards continuous improvement in schools.

The establishment of IQMS therefore does not replace the former strategies (DAS, PMS and WSE), but incorporates them. IQMS emerged as a means to reconcile the three quality management strategies; however, the separate purposes of DAS, PMS and WSE remain intact which seems to be problematic for the harmonious integration of the three policies. This apparent lack of integration therefore does not only undermine the implementation of IQMS towards continuous improvement, but also serves as a major cause for tension between improvement and accountability. In order to prevent IQMS from becoming an 'added on' approach and window-dressing exercise, mechanisms promoting the harmonious integration should therefore be examined.

\section{Theoretical Location of the Study}

Dreyer (2008) asserts that although theory cannot capture the full complexity of life, it does offer frameworks for understanding and interpreting experience and suggests particular courses of action. As this study intends to develop a framework for a model that fulfils the original aims of IQMS as a culture for continuing improvement, the development of such model cannot be conducted successfully without placing it within a specific context or framework and considering the complexities between the model as a system and other systems within and outside the school system as a whole. According to Green (2001) teachers, principals, parents, learners and policy makers all make sense of their own experiences from particular perspectives. These different perspectives can enrich and contribute to creative and novel solutions to human problems (Green, 2001). Engelbrecht (1999) contends that it is therefore difficult to understand the value and actions of people if seen in isolation, divorced from the social context.

The underlying epistemological assumption of this study is that IQMS is driven by a need for accountability at the expense of development, and therefore undermines continuing self-improvement in schools. People are constantly engaged in constructing meaning that involves formal, intuitive and creative knowledge (Dreyer, 2008). It is thus very important to understand the development of a model for continuing self-improvement in schools as part of the whole school, as part of the community in which the school operates, the broader political and social environment, as well as part of the continuing debate on school improvement and quality assurance practices.

The focus is to critique IQMS and its philosophical underpinnings and to advocate that IQMS be re-invented and used under more refined lines in which the three IQMS policies can truly integrate to support the continuous professional development perspective. The theoretical framework influencing my approach to this study is essentially located within Senge's Systems Thinking. This should help in gaining an understanding and critique of current IQMS practices in schools. This theory is outlined in greater detail below.

\subsection{Systems Thinking}

Although Senge (1990) believes all five disciplines within "The Fifth Discipline, the Art and Practice of the Learning organization" must be addressed, he emphasizes systems thinking because it integrates the disciplines. By systems thinking, Senge is referring to a "body of knowledge and tools" that help us to see underlying patterns that are roadblocks to change, but not specific people or events. Systems thinking is the conceptual cornerstone of Senge's approach, as it is 
viewed as the discipline that integrates others, fusing them into a coherent body of theory and practice. The System's theory ability to comprehend and address the whole and to examine the interrelationship between the parts provides both incentives and the means to integrate the disciplines for Senge. He argues that one of the key problems with much that is written about, and done in the name of management, is that rather simplistic frameworks are applied to complex systems. According to this view, we tend to focus on the parts rather than the whole. An appreciation of systems will lead to recognition of the use of, and problems with, reinforcing feedback, and an understanding of the place of balancing (or stabilizing) feedback.

Senge asserts that the fragmentation that exists in the education process is extraordinary. Part of it is embedded in our theory of knowledge, which according to Senge puts knowledge in cubbyholes. Based on this view, the problem here has to do with very deep issues regarding the fragmentation of knowledge and our incapacity to integrate. Furthermore, Senge believes that unless the system is changed, it will continue to create the same results, despite personal differences, individuals in a given system are likely to behave in similar ways. This theory is applicable in this study that attempts to reconcile the fragmented knowledge of the three IQMS policies. The attempt to re-invent IQMS into an inclusive system, which educators will not view as fragmented, three different policies, but rather one integrated system. The implementation of IQMS as an inclusive system has the potential to enhance the collective capacity of educators to create and pursue the aspect of continuous improvement in schools.

\section{Research Methodology}

\subsection{Design and paradigm}

A case study design was used to describe and understand in a subjective manner the qualitative nature of the phenomenon IQMS, as a tool towards continuous improvement. The interpretive paradigm was used in a suggestive manner to generate a way of re-inventing IQMS towards a culture of continuing self-improvement in primary schools. Insights have therefore been drawn from the interpretive paradigm which is concerned with understanding the reality under investigation. Elements of the interpretive paradigm have been explored in order to ground this study in its philosophical and methodological orientations. Furthermore, this paradigm guided the researcher to use semi-structured interviews to uncover aspects of IQMS that promote and undermine continuing self-improvement in schools respectively. Moreover, according to this perspective, people attach meaning to the various everyday activities they undertake. With the nature of reality dependent on individuals and groups for their form and content according to Naidoo (2009), school leaders and teachers do not only attach different meanings to their activities, but they also have different perspectives on IQMS and the role it fulfills as a culture of continuing improvement in schools.

\subsection{Participants}

The broader population on which this research focuses includes principals, school management team members (SMTs) and post level one teachers in all primary schools in the Buffalo City Metropolitan area. This sample, drawn from the geographical area of the Buffalo City Metropolitan area, is representative of schools from three of the previous education departments (Department of Education and Training, House of Representatives and Cape Provincial Department of Education). One of the reasons for selecting these schools is that they all are information-rich cases, which provided valid knowledge and meaningful insight.

Once I have identified four schools, three educators were selected from the school's staff lists to voluntary take part in the semi-structured individual interview. The principal of each school was selected because of his/her leadership role as the 'driver' of quality assurance and school improvement, whilst one member of the School Management Team (SMT) was selected on the basis of their overall responsibility in overseeing quality assurance practices and procedures. The choice of teachers was based on the understanding that each and every teacher is responsible for undertaking a self-evaluation of his/her personal performance and their professional accountability to ensure and maintain quality teaching and learning.

\subsection{Data Collection}

Semi-structured interviews were conducted with principals, school management team members (SMTs) and post level one teachers, of the purposively selected schools who agreed to participate in the study. The interview schedule consisted of two sections. Section A sought personal information related to background variables such as gender, 
teaching experience and position (rank). Section B focused on aspects related to the implementation and functioning of IQMS and comprised eleven questions, covering different topics to ensure that questions address the same theme in all interviews. Although there were pre-set, open-ended questions for all participants (post level one teachers, principals and SMT members), neither the wording, nor the order of the questions were fixed and the questions followed the lines taken by the interviewees' cues and interests (Creswell, 2002; McMillan \& Schumacher, 2001). The interviews were conducted either in an office in the administration block of the school, or in a classroom to ensure an environment that is free from noise and comfortable to the interviewees. It was explained that the interviews would be conducted in English, but interviewees could respond in any language of their choice, isiXhosa or Afrikaans.

\section{Findings and Discussion}

\subsection{Introduction}

A sound and integrated approach for systematic, continuous improvement, where authority is dispersed, is key towards the enhancement of student learning and performance excellence. Raising the quality of teacher performance through teacher development programmes is essential to improve the overall performance of the education system (Mestry, Hendricks and Bisschoff, 2009).

Based on Senge's argument, the fragmentation that exists in the three IQMS policies can be considered as one of the main reasons for the individualistic implementation and use of these policies. So part of the problem with IQMS for continuous improvement of quality has to do with very deep issues regarding the fragmentation of knowledge and participants' incapacity to really integrate. Systems thinking is being considered as a key factor in transforming schools into learning organizations in which people at all levels are collectively, continually enhancing their capacity to create what they are passionate about and to pursue the overall vision of the school. According to Senge, teachers do not work together, resulting in very little sense of collective learning going on in most schools. With this individual culture that is so deeply embedded in our schools, the fragmented view of IQMS is further reinforced, making it very difficult for teachers to see it as an integrated system. It is therefore critical to consider systems thinking as a discipline that needs to be developed in an attempt to integrate and fuse the three IQMS policies into a coherent body of theory and practice. In order to explore the theoretical compatibility and practical benefits of combining the system dynamics in IQMS, the Systems theory, the overarching theoretical framework in this study, will be used in a suggestive manner.

The discussion of the findings is embedded within the Systems theory which forms the overarching framework for this study. This framework forms the cornerstone of the re-invention approach for IQMS to become a tool for continuous improvement in schools. Furthermore, a culture of continuous improvement is closely linked to the concept of learning organizations. From a theoretical perspective, Senge (1990) refers to the System theory's ability to comprehend and address the whole, and to examine the interrelationship between parts. Although Senge believes all five disciplines must be addressed, he emphasizes systems thinking because it integrates the other disciplines. The focus on individual policies in IQMS is in line with Senge's (1990) argument that one of the key problems is the tendency amongst people to focus on the parts rather than the whole, and their failure to see systems as a dynamic process. Thus, according to this argument, a better appreciation of systems will lead to more appropriate action in terms of continuous improvement in schools.

The discussion of findings illuminates two important themes; however, with the divergence in thinking with regard to IQMS practices and systems, different subthemes emerged. In this section the themes referred to are: tension between IQMS policy and continuous improvement and a proposed framework for the re-invention of IQMS. Each of these aspects is explained in greater detail below.

\subsection{Tension between IQMS Policy and Continuous Improvement}

In this study, despite acknowledging that IQMS has contributed to acts of working together and constant communication and collaboration, these claims seem to be superficial and a form of window-dressing. The IQMS is an ambitious integrated strategy which embraces three systems (developmental appraisal, performance management and whole school evaluation), aiming to improve quality in South African schools. However, although IQMS views itself as an integrated tool, an analysis of the findings shows that it has not been the case, as each of these programmes fulfills a discernible function. In spite of the name of the policy which seeks to foreground integration, the findings underscore the perception that the three policies of IQMS do not integrate properly. This suggests that the tension between these different approaches subvert the development and continuous improvement that has hitherto taken place. 
An inference that can be made is that claims of 'collaboration' and 'working together' are not for the sake of quality improvement and school improvement in general, but rather for the sake of camaraderie, salary progressions and bureaucratic accountability. Furthermore, the findings indicate that there is a disconnection between the improvement of quality for which IQMS was designed and implemented, and the purposes for which IQMS is presently used by educators. Based on this view, the findings point to an interesting dynamic on how this is manifested within schools.

First, the integration of DAS and PMS has resulted in a tug of war between these two systems. With no visible or tangible rewards attached to DAS, no importance is being attached to teacher professional development, which in practice unfortunately often deteriorates into a mechanical and meaningless exercise. Second, the reward system attached to PMS inevitably forces teachers to hide areas of weakness, thus concealing all aspects of development and continuous improvement. Considering the separation of these three policies, it would therefore seem that IQMS, despite its noble intensions, will never realize the third component of whole school evaluation which involves improvement of the whole school through a structured school improvement plan.

In addition to the tension between the three IQMS policies, lack of coherence and interactivity between certain internal and external practices in IQMS causes tension which results in this process being viewed with markedly less positive attitudes amongst educators. Constraints contribute to lack of capacity because of the tension between policy and development and the focus on incentives rather than quality.

\subsection{Tension between policy and development}

Associated with the theme of development, educators pointed to the tension between IQMS policy and development. Although IQMS was developed and introduced in schools as an integrated system aimed at quality improvement and development, findings indicate that there is a disconnection between improvement and quality of teaching and learning and the definition of quality assurance described in the purpose and guiding principles of the IQMS policy. In addition, the notion of 'quality improvement' is considered problematic, because the focus is not on improving quality, but rather on complying with predetermined performance standards, which are geared towards external ownership leading to compliance instead of improvement. In other words, IQMS is used for compliance with external requirements, which may pass for improvement in the short term, but as soon as the need to display 'improvement' has passed, old habits are likely to re-emerge. Based on these perspectives, it is obvious that IQMS for improvement purposes, which implies a formative approach that does not focus on control, but on improving quality, is being undermined.

\subsection{Dependence on external control}

The management culture embedded in IQMS finds expression in an external quality assurance exercise which introduces a new bureaucracy and increased centralized management controls. IQMS has made it mandatory for educators to account to their appraisers and this undoubtfully supports management in monitoring the work of educators, and hence compels teachers to be accountable for their actions. The most compelling indicator of this external managerial culture is the introduction of a new bureaucracy and increased centralized managerial control. This quality assurance perspective therefore appears to support the centralization of managerial control and by implication, tactically strengthens the managerial culture within the schools to the Department of Education.

\subsection{Focus on incentives rather than quality}

The initiation of a monetary incentive is viewed as a serious drawback that inevitably creates resentment amongst participants. When IQMS is viewed in this light, the improvement aspect is further undermined, as it creates a culture of self-interest for financial reward which takes precedence over development and improvement. Moreover, the direct link to funding serves as an incitation to hide weaknesses, manipulate data and the creation of a compliance culture according to Kis (2005). The implication of a quality assurance system that is being driven by rewards does not only reduce internal motivation, but the drive for monetary rewards completely subsumes the developmental agenda of IQMS. When a quality assurance system is viewed through this perspective, it leads to lack of intrinsic enthusiasm and impetus for the process, which further negates the improvement and empowering capacity of the instrument.

All these constraints call for the re-invention of IQMS. However, in order to re-configure IQMS, and to release the tension between improvement and policy, it was necessary to understand the practices and systems that contributed to their irritations and frustrations, but nevertheless result in a palpable alteration of structures of action. The purpose of IQMS is the enhancement of continuous quality development in schools, which is achievable if an appropriate alignment 
can be found between IQMS philosophy and improvement. The alignment sought through this re-invention process is between the three IQMS policies, a quality culture and external and internal requirements.

In the light of the findings gleaned from this study, and the theoretical framework. I would like to propose a framework for the re-invention of IQMS towards a culture of continuous improvement.

The re-configured IQMS should: (1) Look for mechanisms to integrate the three policies, making lines of articulation between the three policies clear, and where accountability imperatives do not conceal the developmental needs of educators. (2) Develop systems thinking that integrates the different parts.

\section{Proposed IQMS Framework for Continuous Improvement}

It became clear that due to various constraints to continuous improvement, IQMS has not achieved the objectives for which it was originally designed. The following section looks at the re-invention of IQMS as a tool for continuous improvement. I propose the following framework so that IQMS represents a culture for continuous improvement. The framework has two purposes: The first one is to use it as a tool to critique the current conceptualization and practice of IQMS. The second part provides the basis for a possible re-invention. I provide a framework that is based on three pillars namely: IQMS as an integrated system; clarity of purpose within an integrated quality assurance instrument; and the decentralization of IQMS.

It is against this background that the re-configured IQMS should: (1) Look for mechanisms for integrating the three policies that will make lines of articulation between the three policies clear, and where accountability imperatives do not conceal the development needs of educators and the school; and (2) Development of systematic thinking that integrates the different parts. In a similar vein, the re-invention and development of IQMS to underpin the construction of a successful quality culture for continuous improvement, thus requires:

\subsection{IQMS as an integrated system}

With IQMS designed as an integrated quality assurance system, the assumption is that the three policies are integrated. With a 'system' commonly referring to a group of linked parts that work together toward a common end, the three different parts of IQMS have been integrated with a coherent set of practices and goals. Despite IQMS seeking to amalgamate DAS, PMS and WSE, focusing primarily on the work performance of educators as the core of these quality assurance initiatives, there can be little doubt that appraisal/evaluation/measurement are not used in a holistic way, but rather viewed as separate instruments. The focus of an integrated system should therefore be on quality education for all, transformation and advantages for educators, schools and the system as a whole. It should also address the relationship between the three programmes as to how they should inform and strengthen one another in an integrated system (IQMS Manual, 2005 Section B: 20). While the whole school development initiative can not be seen as separate or distinct to the learning and empowerment of the individual educator, confusion still exists with respect to appraisal, evaluation and measurement.

Evidence shows that the three IQMS policies are not viewed as integrated and therefore not seen as holistically interdependent and interlinked, fostering the process of improving the 'whole school'. The simultaneous application and development of the three policies are therefore essential to encourage a sense of 'wholeness' amongst educators. The complexity of identifying different systems within the so-called integrated system casts further doubt on the integration between the three policies. As far as IQMS is concerned, no direct mention of evaluation paradigms or variables can be found. Instead this system needs to be seen and applied as a whole, making use of performance standards where the standard is like a "flag that sometimes distinguishes one cavalry regiment from another one" (Hariparsad, Bisschoff, Conley, du Plessis, Grobler, Hlongwane, Loock and Mestry, 2008).

For any re-invention process particularly IQMS to be successfully implemented, it is necessary to be aware of the ambivalent character of IQMS in relation to the existing tension and ambivalence between accountability and improvement. According to Vroeijenstijn (1995b), the purpose of accountability is incompatible with the purpose of improvement. Based on this view and in addition to the lack of integration, there is also a form of duality in IQMS as it is concurrently centralized and decentralized. The Government and Department of Education attempt to decentralize power by giving autonomy and devolving responsibility to schools; however, fear of declining standards could perhaps be the reason for centralizing the system through accountability requirements. 


\subsection{Clarity of purpose within an integrated quality assurance instrument}

Expectations regarding the aims and outcomes of IQMS seem to differ between different stakeholders (educators and Department of Education), hence in order to create a coherent system, the aims of the integrated quality assurance system must be clear, with no contradiction between any of them. From this study, it was found that each of the three IQMS policies had been implemented with a distinct focus and purpose, with the main motives of educators focused on financial rewards, and the Department of Education mainly interested in accountability, which suggests no clarity of purpose. In the process of re-inventing IQMS for continuous improvement, quality should be enhanced more easily through improvement approaches than through control. In order to enhance clarity of purpose within an integrated quality assurance instrument, improvement and accountability must be conceptually and practically distinct, with separate resourcing, while allowing for close contact between them. Furthermore, this would also call for comprehensiveness in a quality assurance system that is consistent with the mission and core values of the school.

\subsection{The decentralization of IQMS}

According to McGinn and Welsh (2009), governments actually ran schools in most countries, while in a few countries they monitored schools run by non-governmental groups or private individuals. The transfer of educational decision-making authority and responsibility from the center to regional and local systems has become an increasingly popular reform in many countries. In order to strengthen my argument for the re-invention of the quality assurance system to one that is internally driven and owned by all stakeholders in schools, the process of decentralization is proposed. Bradshaw and Buckner (1994) believe that the significant changes demanded of schools can only be attained through such devolution of power that encourage people to change and to address educational problems. The policy framework for decentralized decision making, which paved the way for school-based management, is furthermore embedded in the SA Schools Act 84 of 1996 (South Africa 1996). The re-invention of IQMS could therefore be initiated through decentralization, which allows for the re-construction of IQMS from a bureaucratic, externally driven system, to one that is internally driven, shifting the location of power and authority from those in one location (e.g. Department of Education) to those in another location (e.g. schools).

\section{Concluding Remarks}

It can be concluded that the current conceptualization and practices of IQMS do not necessarily translate into continuous quality assurance improvement in schools. The dilemma is as a result of the three policies that are implemented and used as fragmented programmes, with diverse goals, multiple purposes, values that are at variance, and conflicting expectations, causing tension between personal needs and improvement needs of educators. Issues of continuous quality assurance improvement are hampered by external and internal quality assurance practices that are under managerial imperatives of compliance and accountability. The re-invented IQMS provides us with the possibility for a quality assurance management tool that can provide continuous improvement in schools.

\section{References}

Biputh, B. \& McKenna, B. ( 2009).Tensions in the quality assurance process in post-apartheid South African Schools. Journal of Comparative and International Education.

Creswell, J. ( 2002). Research design, Qualitative and Quantitative Approaches. Thousand Oaks, CA: Sage.

De Clercq, F. ( 2008). Teacher quality, appraisal and development: The flaws in the IQMS. Perspective in Education 26(1), 7-17.

Department of Education ( 2009). 2009/10 Ministerial Annual Report on Education. Pretoria: Government Printer.

Department of Education (1996). Towards quality education in general education and training, Policy brief 1. Pretoria: Government Printer.

Department of Education (1996). South African Schools Act 84 of 1996. Pretoria: Government Printer.

Department of Education (2005). Integrated Quality Management System (IQMS) for School-Based Educators. Pretoria: Government Printer.

Dreyer, L. ( 2008). An evaluation of a model for learning support in primary schools in the Westcoast-Winelands region. PhD Dissertation. Stellenbosch: University of Stellenbosch.

Education Labour Relations Council (ELRC) ( 2003). Collective agreements 3 (Protocol) and 8, Integrated Quality Management System.

Engelbrecht, P. (1999). A theoretical framework for inclusive education. In P. Engelbrecht et al. (eds.) Inclusive education in action in South Africa. Pretoria: Van Schaik. 
Gardner, M. (2003). Continuous Quality Assurance Management. Queensland: University of Queensland.

Green, L. (2001). Theoretical and contextual background. In P. Engelbrecht \& L. Green (eds.) 2001. Promoting Learner Development: Preventing and working with barriers to learning. Pretoria: Van Schaik.

Hariparsad, I. D.; Bisschoff, T. C. B.; Conley, L. N.; du Plessis, P.; Grobler, B. R.; Hlongwane, S.; Loock, C.; Mestry, R. ( 2008). Quality assurance in South African schools: An integrated research report.

Harvey, L. (2002). The End of Quality? Quality in Higher Education, Vol. 18, No 1.

Kis, V. (2005). Quality Education in Tertiary Education: Current Practices in OECD Countries and a Literature Review on Potential Effects. France: Education and Training Policy Division: Directorate of Education.

MacMillan, J. H. \& Schumacher, S. ( 2001). Research in Education: A conceptual introduction (5th Edition). New York: Longman.

McGinn, N. \& Welsh, T. ( 1999). Decentralization of Education: Why, when, what and how? Paris: United Nations Educational Scientific and Cultural Organization. Series: Fundamentals of educational planning, 64, IIEP-UNESCO.

Mestry, R.; Hendricks, I. \& Bisschoff, T. ( 2009). Perceptions of teachers on the benefits of teacher development programmes in one province of South Africa. Journal of Education, Vol 29, No.4.

Naidoo, D. (2009). Organizational culture and external quality assurance, PhD thesis. Stellenbosch: University of Stellenbosch.

Rabichund, S. (2011). The Contibution of the Integrated Quality Management System to Whole School Development. PhD thesis. Pretoria: University of South Africa.

Senge, P. (1990). The Fifth Discipline. The art and practice of the learning organization. New York: Doubleday.

Vroeijenstijn, L. (1995b). Improvement and Accountability: navigating between Scylla and Charybdis. Higher Education Policy Series 30.

Weber, E. ( 2005). New controls and accountability for South African teachers in schools: The Integrated Quality Management System. Perspective in Education, 23(2), 63-72. 